\title{
Comparison of Ultrastructure of Germinating Pea Leaves Prepared by High-Pressure Freezing-Freeze Substitution and Conventional Chemical Fixation
}

\author{
Yasuko Kaneko and Paul Walther* \\ Department of Regulation Biology, Faculty of Science, Saitama University, Urawa, 338 Japan \\ -Laboratory for Electron Microscopy I, Federal Institute of Technology, CH-8092 Zürich, Switzerland
}

\begin{abstract}
High-pressure freezing-freeze substitution (HPF-FS) methods were applied to germinating pea leaves. Good ultrastructural preservation without visible freezing damage was obtained up to $200 \mu \mathrm{m}$ in thickness. Compared to conventional chemical fixation (CF), cellular membranes were smoother without undulation, and organelles appeared more turgid. The matrices of cytoplasm and organelles were denser and more homogeneous. These features imply that HPF-FS samples retain more substances and ultrastructure closer to the living state. There were differences in membrane stainability among organelles in HPF-PS specimens, which were not seen after CF. Bundles of microfilaments were observed frequently after HPF-FS.

Key words: high-pressure freezing, freeze substitution, cryofixation, chemical fixation, Pisum sativum
\end{abstract}

By cryofixation, it is expected to be possible to observe ultrastructures closer to the living state of biological specimens, than by chemical fixation $(\mathrm{CF})$ which is known to induce many artifacts during the fixation process. ${ }^{1)}$ However, application of cryofixation to biological specimens has been limited because, under atmospheric pressure, only a very restricted area of biological specimens (up to $10-20 \mu \mathrm{m}$ from the surface) can be frozen satisfactorily. ${ }^{2)}$ High-pressure freezing (HPF) was introduced to overcome this limitation. ${ }^{2)}$ By applying a pressure of $210 \mathrm{MPa}$, it is possible to freeze biological specimens of up to several hundred micrometers in thickness in good condition without using a cryoprotectant. $^{2)}$ By applying high-pressure freezing-freeze substitution (HFP-FS) techniques to plant specimens, novel ultrastructural images were obtained in root tips, ${ }^{3,4)}$ root nodules, ${ }^{5.6)}$ and tentacle stalk cells, ${ }^{7)}$ providing more realistic ultrastructural information than conventional $\mathrm{CF}$.

In this study, HPF-FS techniques were applied to germinating pea leaves, and the ultrastructural details were compared with those obtained by conventional CF.

\section{MATERIALS AND METHODS}

\section{Plant materials}

Seeds of Pisum sativum L. were imbibed in water for $6 \mathrm{~h}$ to overnight at room temperature. Plumules were excised with a razor blade and either subjected to highpressure freezing or fixed by conventional chemical fixation.

\section{High-pressure freezing and freeze substitution}

The plumules were sliced to a thickness of approximately $0.2-0.4 \mathrm{~mm}$ with a razor blade and placed between specimen holders (Bal Tec, BU $012125 \mathrm{~T}$ ). The air space was filled with a drop of 1-hexadecene. ${ }^{8)}$ The specimens were frozen in a high-pressure freezing apparatus (Balzers, HPM 010) and transferred into acetone containing $2 \%$ osmium tetroxide for freeze substitution. The substitution was carried out at $-90^{\circ} \mathrm{C}$ overnight, at $-80^{\circ} \mathrm{C}$ for $5-6$ days, at $-60^{\circ} \mathrm{C}$ overnight and at $-30^{\circ} \mathrm{C}$ overnight. Specimens were brought up to room temperature and embedded in Spurr's resin.

\section{Chemical fixation}

Specimens were fixed in $2 \%$ glutaraldehyde in $0.05 \mathrm{M}$ potassium-phosphate buffer $(\mathrm{pH} 7.0)$ for $1 \mathrm{~h}$ at room temperature and in a refrigerator $\left(4^{\circ} \mathrm{C}\right)$ overnight. After rinsing with the buffer, the specimens were postfixed with $2 \%$ osmium tetroxide in the same buffer for $2 \mathrm{~h}$ at room temperature, dehydrated in an acetone series, and embedded in Spurr's resin.

\section{Electron microscopy}

The ultrathin sections of both HPF-FS and chemically fixed samples were stained with $2 \%$ uranyl acetate for $10 \mathrm{~min}$ followed by lead citrate for $5 \mathrm{~min}$. They were observed at $100 \mathrm{kV}$ in a Hitachi $\mathrm{H}-700 \mathrm{H}$ electron microscope.

\section{Light microscopy}

Thick sections (ca. $500 \mathrm{~nm}$ ) were cut from the same block as for electron microscopy and stained with $0.1 \%$ toluidine blue $\mathrm{O}$ in $1 \%$ borax.

\section{RESULTS}

Segments of pea plumules were frozen without visible ice crystal damage up to about $0.2 \mathrm{~mm}$ in thickness (Figs. 1 and 2). Cracks in cytoplasm and cell walls were occasionally observed especially at the outer layer of the 
specimens (Fig. 3). The percentage of area with good ultrastructural preservation varied somewhat with each freezing and on average corresponded to $30-50 \%$ of sections.

All intercellular membranes were smooth and the organelles appeared turgid in HPF-FS samples. The matrices of cytoplasm and organelles were denser and more homogeneously distributed, implying that more substances were retained in the HPF-FS samples. With chemical fixation, plasma membranes were often undulated and there was usually some space between the cell wall and the plasma membrane (Fig. 4), but with HPF-FS material, plasma membranes were very smooth and appeared as straight lines closely appressed to the cell wall (Fig. 5). No space was observed between the cell wall and the plasma membrane. Boundary membranes of lipid bodies were also smooth and more clearly observed with HPF-FS (Fig. 5) compared to CF (Fig. 4).

The Golgi apparatus exhibited more dynamic structural features in HPF-FS (Fig. 8) compared to CF (Figs. 6 and 7). Each cisterna was more distinctively separated and a clear gradation of membrane stainability was observed from cis to trans face in HPF-FS specimens. Forming vesicles were more easily observed and intercisternal elements (Fig. 8, arrows) were seen in HPF-FS materials.

Mitochondrial membranes were smooth without undulation and were conspicuous in HPF-FS because they were more intensely stained than the membranes of other organelles. Both outer and inner envelope membranes exhibited very similar electron densities, and they were closely appressed to each other so that the space between the two membranes was often difficult to observe.

The matrices of mitochondria and plastids were more homogeneously distributed in HPF-FS (Figs. 10 and 12) compared to CF (Figs. 9 and 11). Although with CF, envelope membranes of mitochondria and plastids exhibit very similar appearances (Fig. 11), in HPF-FS materials there were very distinct differences between them (Figs. 10 and 12). Unlike mitochondrial membranes which stained very intensely, plastid envelope membranes were often much fainter and more difficult to observe. There was also a difference in stainability between outer and inner envelope membranes of plastids; often inner membranes were even fainter and more difficult to observe (Figs. 12 and 14, small arrows). There was a wider space between outer and inner envelope membranes of plastids compared to mitochondrial envelope membranes.

Nuclear membranes were also difficult to observe in HPF-FS samples (Fig. 14). However when they were visualized, they appeared as parallel straight lines and the widths across the two membranes were wider than in CF materials. The nuclear matrix was filled with flocculent substances which were distributed mor $\epsilon$ ivenly in HPF-FS (Fig. 14) than CF (Fig. 13). ER membranes were often associated with plastids and mitchondria in these specimens (Figs. 2,11, 12 and 13). However, they were often difficult to observe in HPF-FS because of the low electron density. The appearance of ER membranes (Fig. 12) was similar to nuclear envelope membranes (Fig. 14) in HPF-FS specimens. They also appeared as parallel straight lines and the width across the two membranes was wider (Fig. 12) than in CF materials (Fig. 11).

Bundles of microfilaments were very frequently encountered in HPF-FS materials (Fig. 15) but rarely seen in CF materials. They were often associated with the ER (Fig. 15).

\section{DISCUSSION}

By applying HPF-FS methods to germinating pea leaves, well reproducible ultrastructural preservation was obtained. The general appearance of the ultrastructural features was similar to that of other plant specimens prepared by HPF-FS. ${ }^{3-7)}$ We assume, that by HPF-FS the cells were fixed closer to the living state than with CF because the membranes appear smoother and the cytoplasm appears denser. This interpretation implies that by $\mathrm{CF}$ the originally dense matrix is partially washed out (e.g. during dehydration) and that the originally smooth membranes become undulated during CF. It would be very unlikely, that originally undulated membranes would become smooth and that the matrix would become denser

Fig. 1. Lght micrograph of a mid-transvers section of a thssue block of pea plumule subjected to HPF-FS. 1: primary leaf; 2: secondary leaf; 3: 3rd leaf; 4: 4 th leaf.

Fgs. 2 and 3. Fectron micrugraphs oí a section adjacent to Fig. 1. A central portion (Flg. 2) and a peripheral portton (Fig. 3) of a 3rd leaf. No ice crystal damage is observed throughout the section. Cytoplasmic cracks are observed in epidermal cells (E) in Fig. 3 . M: mitochondrion; N: nucleus; P: plastld; PB: protein body.

Figs. 4, 6, 7 and 9. Portions of 3rd leaf celts of pea plumule prepared by conventional CF.

Figs. 5, 8 and 10. Portlons of 3rd leaf cells of pea plumule prepared by HPF-FS.

Figs. 4 and 5. Piasma membrane (PM) and liptd bodies (L). Plasma membrane in Fig. 5 appears as straight lines and tightly appressed to cell wall. $\mathrm{CW}$ : cell wall.

Figs. 6, 7 and 8. Golgi apparatus (G). Intercisternal element (arrows) is observed in Fig. 8. M: mitochondrion; PB: proteln body.

Figs. 9 and 10. Mitochondria (M). Envelope membranes are intensely stained and tightly appressed to each other in Fig. 10 . PB: protein body.

Figs. 11 and 13. Portions of 3rd leaf cells of pea plumule prepared by conventional CF.

Flgs. 12, 14 and 15. Portions of 3rd leaf cells of pea plumule prepared by HPF-FS.

Figs. 11 and 12. Plastids (P) with assoclated ER. Inner envelope membrane (arrow) of plastid is faintly observed in Fig. 12 . M: mitochondrton. Flgs. 13 and 14. Portions of nuclel $(N)$ and plastids $(P)$. Nuclear envelope membranes (large arrow) show up as straight lines in Fig. 14. Small arrow points to inner envelope membrane of plastid in Fig. 14. ER: endoplasmic reticulum.

Fig. 15. A bundle of microfilaments (MF) and ER. 

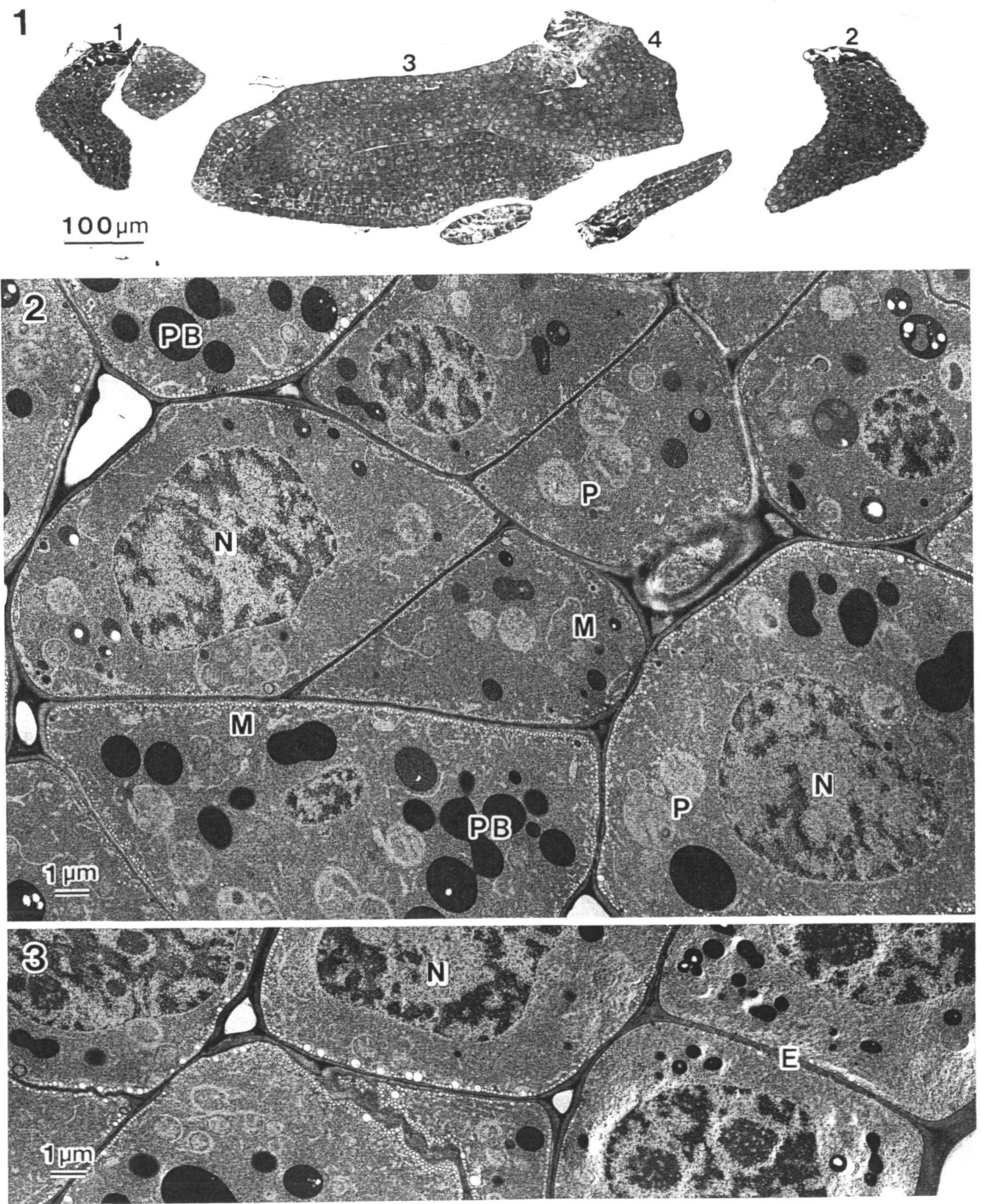

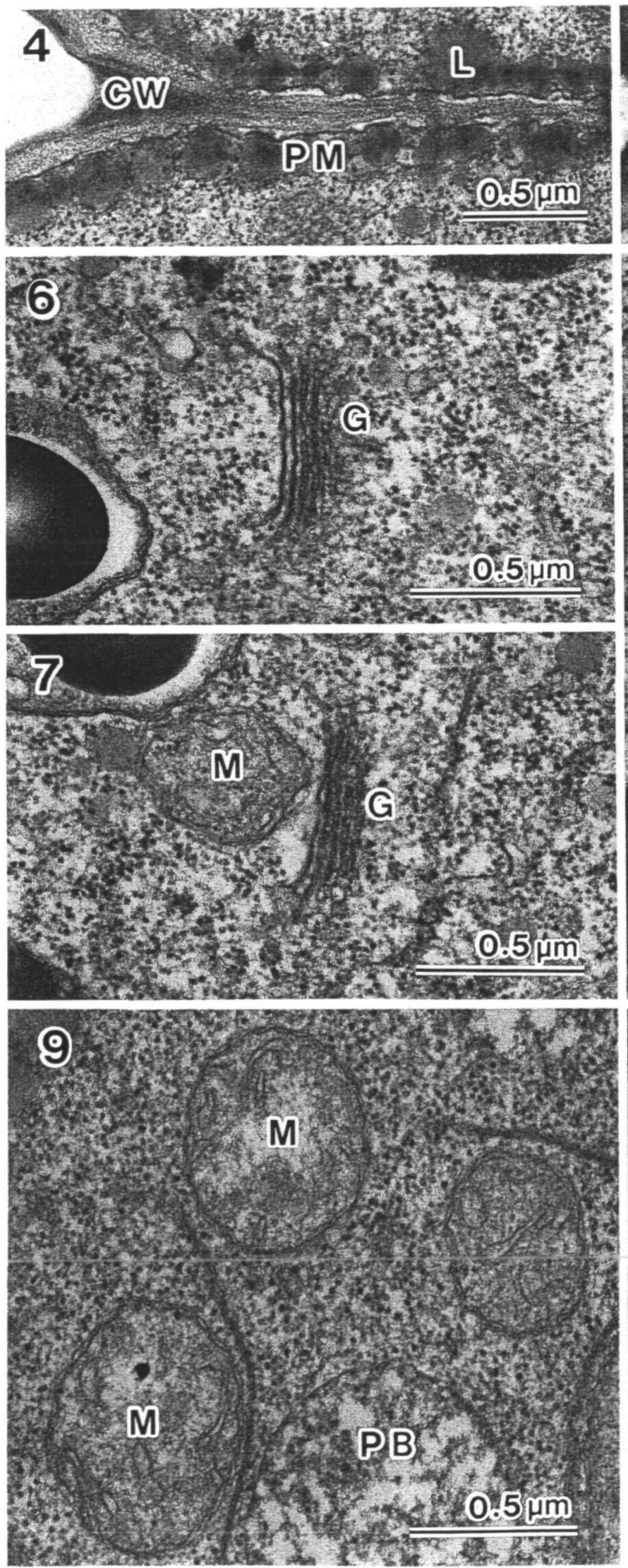
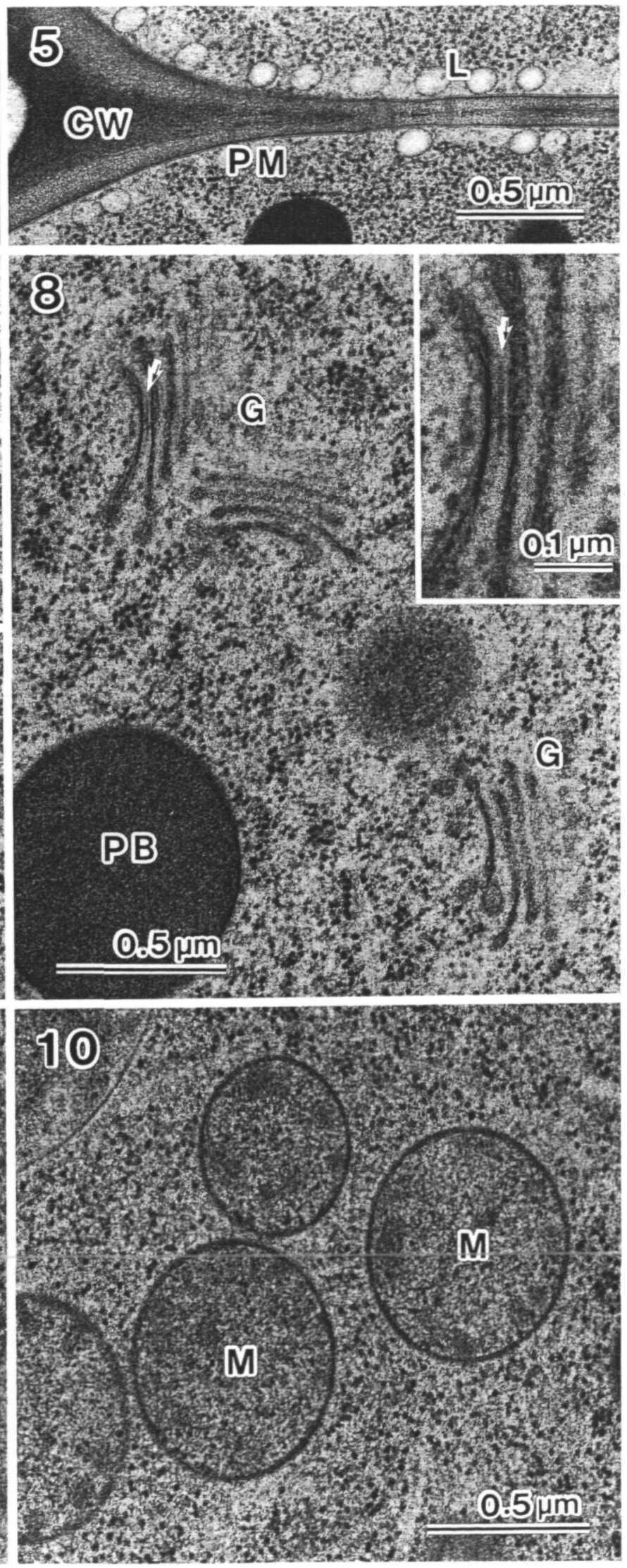

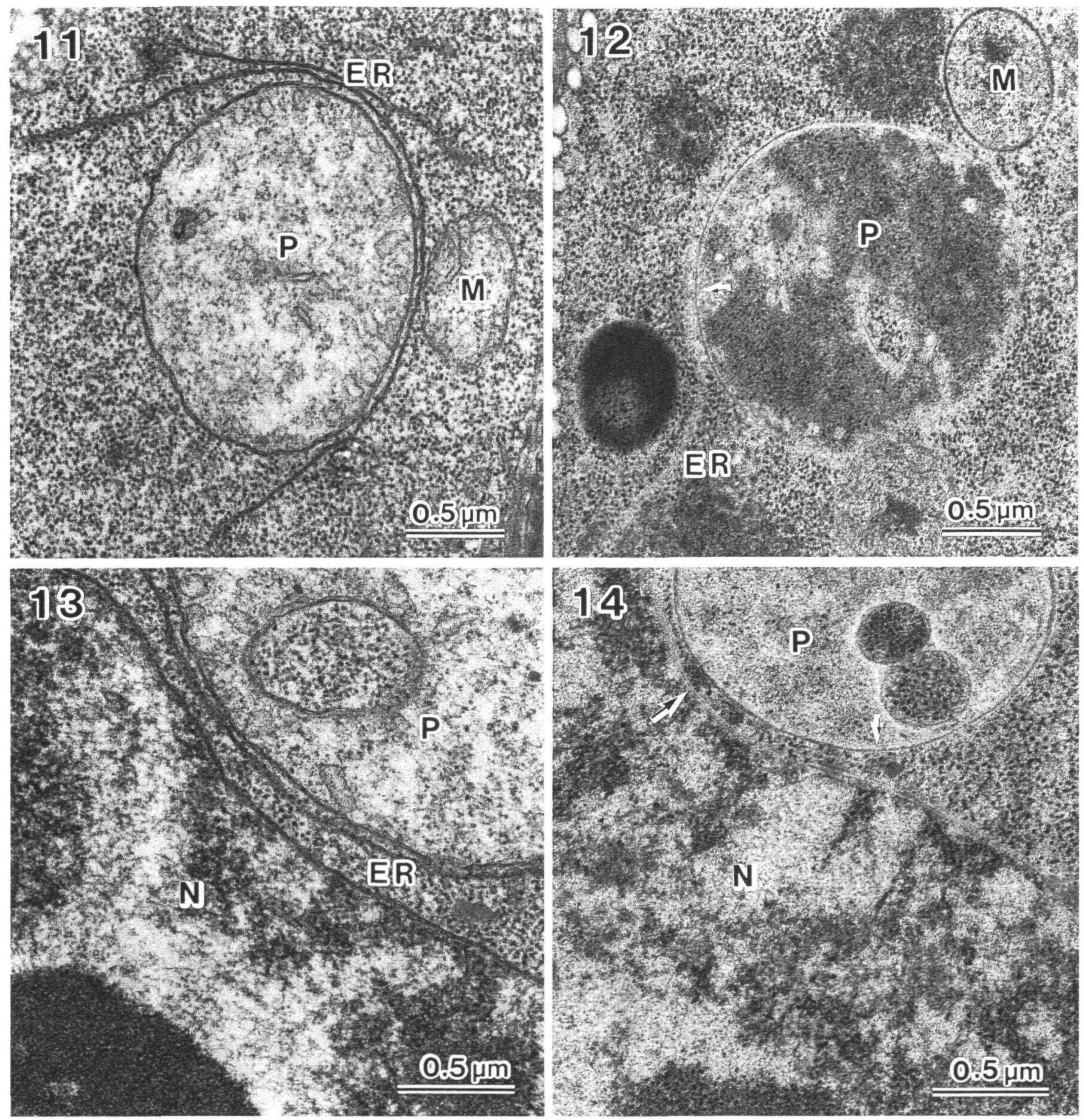

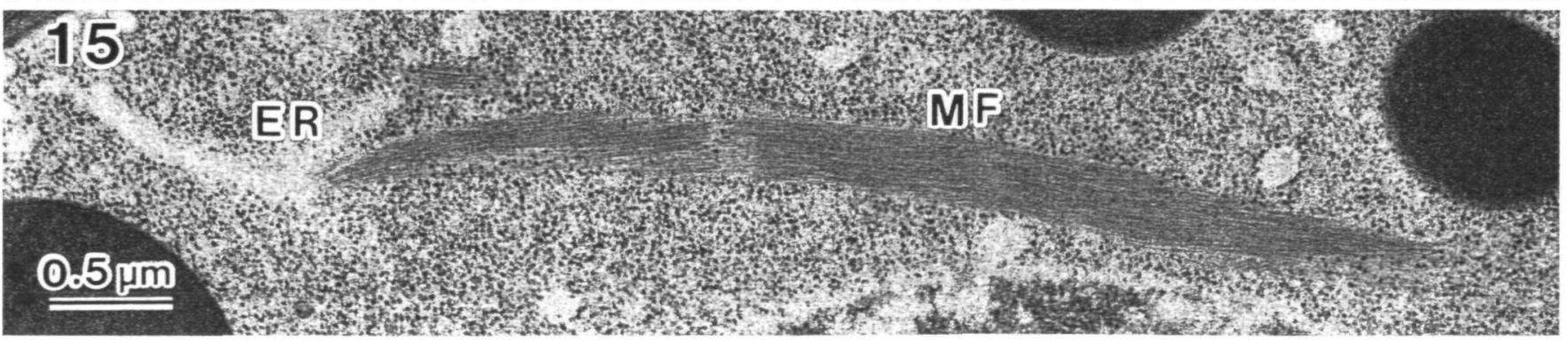


during HPF-FS. HPF is a purely physical process which immobilizes the tissue within milliseconds and is much faster than $\mathrm{CF}$ which takes minutes to act and leads to structural distortion even at the light microscopic level ${ }^{1)}$ and fine structural change caused by uncontrollable osmotic effects. ${ }^{6)}$

The observation that inner and outer mitochondrial membranes were appressed to each other was also made with other cryo-fixation techniques. ${ }^{9)}$ The different appearance of the membranes of mitochondria and plastids after HPF-FS, such as stainability and intermembrane spaces, may indicate a significantly different nature of these membranes. Because electron density of nuclear envelope and ER membranes as well as plastid envelope membranes was very low after HPF-FS, they were often difficult to observe. To follow the active movement and function of these membranes, it would be important to improve the visibility of these membranes, which might be possible by modifying the freeze substitution and poststaining procedures. The Golgi apparatus exhibited a much more lively configuration in HPF-FS specimens, and a clear gradation of membrane stainability across Golgi stacks existed as in Nicotiana root cap cells. ${ }^{4)}$ Intercisternal elements which were previously found only in mucous-producing cells ${ }^{4)}$ were also observed in these specimens after HPF-FS.

One of the prominent features of the specimens prepared by HPF-FS was the preservation of abundant bundles of microfilaments. In CF specimens, the amount and the frequency of such filaments was far less. These filaments are probably destroyed during chemical fixation. Although the nature of the microfilaments has yet to be determined, it is possible that these filaments are involved in the intercellular movement of organelles during germination. Further study is needed to elucidate the function and nature of these filaments.

In this study, HPF-FS was successfully applied to freeze plant specimens of up to about $0.2 \mathrm{~mm}$ in thickness. However, the performance of the freezing was variable with each trial. This variability may have been introduced during specimen preparation before they were applied to the high-pressure freezer, since it was difficult to excise the specimens in a size that fits the specimen holders exactly. Some structural damage such as cracks in cytoplasm and cell wall was observed especially at the outer layer of specimens, which may have been caused by mechanical force during high-pressure freezing. Except for this obvious damage, the effect of applied high pressure on the ultrastructural details was difficult to assess. There is still a possibility that the fine structure of membranes, lipid-protein interactions, or other parts of ultrastructure may be altered because of the high pressure. ${ }^{10)}$ Continued careful examination and assessment is still necessary to further apply these methods for fine structural analysis of biological specimens. In spite of some uncertainties, the potential and capability of freezing thick biological specimens without ice crystal damage for ultrastructural study is outstanding and many novel features of biological specimens are expected to be revealed by further application of HPF-FS methods.

Acknowledgments. We thank Dr. Ya Chen for technical assistance and Dr. Tammo Retsewitz for critlcal reading of the manuscript. High-pressure freeding and freeze substitution were performed at Integrated Microscopy Resource (IMR) in Madison. Wisconsin. The IMR is funded as an NIH Blomedical Research Technology Resource (RR570).

\section{REFERENCES}

1) Mersey B, McCully M: Monitoring of the course of fixation of plant cells. J Microsc 114: 49-76 (1978)

2) Moor $\mathrm{H}$ : Theory and practice of high pressure freczing. In: Steinbrecht RA, Zlerold $\mathbf{K}$, eds. Cryotechniques in biological electron microscopy. Berlin, Heidelberg: Springer-Verlag, 175191 (1987)

3) Kiss JZ, Giddings TH, Staehelin LA. Sack FD. Compartson of the ultrastructure of conventionally fixed and high pressure frozen/freeze substituted root tps of Nicotiana and Arabidopsls. Protoplasma 157: 64-74 (1990)

4) Staehelin LA, Giddings TH, Klss JZ, Sack FD: Macromoleculer differentlation of Golgi stacks in root tips of Arabldopsis and Nicotiane sedlings as visualized in high pressure frozen and freeze-subsututed samples. Protoplasma 157: 75-91 (1990)

5) Newcomb W, Jackson S, Racette S, Torrey JG: Ultrastructure of infected cells in the actinorhizal root nodules of Gymnostoma papuanum (Casuarnaceae) prepared by high pressure freezing and chemlcal fixation. Protoplasma 157: 172-181 (1990)

6) Studer $D$, Hennecke $H$, Müller M: High-pressure freedng of soybean nodules leads to an improved preservation of ultrastructure. Planta 188: 155-163 (1992)

7) Luchtscheidl IK, Lancelle SA, Hepler PK: Actln-endoplasmic reticulum complexes in Drosera. Their structural relationship with the plasmalemma, nucleus, and organelles in cells prepared by high pressure freedng. Protoplasma 155: 116-126 (1990)

8) Studer D, Michel M, Müller M: High pressure freezing comes of age. Scanning Mlcrosc, Suppl 3: 253-269 (1989)

9) Knoll G, Verkleij AJ. Plattner H: Cryofixation of dynamic processes in cells and organelles. In: Steinbrecht RA. Zerold K, eds. Cryotechniques In biological electron microscopy. Berlin, Heidelberg: Springer-Verlag, 258-271 (1987)

10) Dahl R, Staehelin LA: High-pressure freezing for the preservation of blofogical structure: Theory and practice. J Electron Microsc Tech 13: 163-174 (1989)

(Received January 23, 1995; accepted February 28, 1995) 